\title{
A Self-Adaptive and Self-Learning Methodology for Wireless Intrusion Detection using Deep Neural Network
}

\author{
R. Sathya ${ }^{\text {a }}$, Jatin Agrawal ${ }^{b}$, Debayan Roy ${ }^{c}$ and Riddhi Dutta ${ }^{d}$ \\ ${ }^{a}$ Assistant Professor, Department of Computer Science and Engineering, SRM Institute of Science \\ Technology, Chennai, Tamil Nadu, India. \\ ${ }^{\mathrm{b}}$ Department of Computer Science and Technology, SRMIST, Chennai, Tamil Nadu, India. \\ ${ }^{c}$ Department of Computer Science and Technology, SRMIST, Chennai, Tamil Nadu, India. \\ ${ }^{\mathrm{d}}$ Department of Computer Science and Technology, SRMIST, Chennai, Tamil Nadu, India.
}

Article History: Received: 10 November 2020; Revised 12 January 2021 Accepted: 27 January 2021; Published online: 5 April 2021

\begin{abstract}
Cyber physical systems combine both the physical as well as the computation process. Embedded computers and systems monitor to control the physical forms with feedback loops which have an effect on computations and contrariwise. A vast number of failures and cyber-attacks are present in the cyber physical systems, which leads to a limited growth and accuracy in the intrusion detection system and thus implementing the suitable actions which may be taken to reduce the damage to the system. As Cyber-physical systems square measure but to be made public universally, the applying of the instruction detection mechanism remains open presently. As a result, the inconvenience is made to talk about the way to suitably apply the interruption location component to Cyber physical frameworks amid this paper. By analysing the unmistakable properties of Cyber-physical frameworks, it extraordinary to diagram the exact necessities 1st. At that point, the arranging characterize of the intrusion discovery component in Cyber-physical frameworks is introduced in terms of the layers of framework and particular location procedures. At long last, a few imperative investigation issues unit known for edifying the following considers.
\end{abstract}

Keywords: Intrusion Detection Systems, Wireless Network Security, Cyber Security.

\section{Introduction}

With the new technologies and ways being enforced in wireless networks, there have been loads of intrusion problems. to beat this the new firewall and protection systems are enforced, however, the options of the systems aren't abundant effective and aren't capable of overcoming all the new ways that of intrusions. For this several new ways square measure being enforced to achieve the foremost secure and correct intrusion interference and detection system. however, thanks to new updates within the technologies these systems also are on the verge of obtaining negative and comparatively less accuracy within the detection and interference. to cut back on these new Machine Learning techniques square measure being enforced with a colossal variety of algorithms to review all the ways that of intrusion systems and to achieve the high ability of the techniques. With the implementation of the new computing ways within the Intrusion detection system, detection ways supported AI became one of all the hotspots of IDS analysis. The foremost ordinarily used AI ways embody neural network, genetic algorithm and immune algorithm like intrusion detection supported deep back propagation neural network, cooperative intrusion detection supported systems. However, this technique is littered with the loss of key information throughout the tactic of data set, that finally ends up into "distortion" of the sample information, inability of data feature extraction, then it contributes to greater volatility of the take a glance at results. Compared to alternative mil ways, the wireless intrusion detection methodology employing a CNN considerably improves accuracy of classification.

Many sorts of analysis have improved sample recognition abilities and performances. The model that is being enforced these days is that the CNN. The newest ICNN satisfies most of it and has accuracy beyond the older versions. However, this technique has drawbacks that may result in higher false detection rates and may lead to the failure of the detection of the intrusion. the most important drawbacks of this square measure that it's susceptible to three major and large attacks that square measure DOS attack, Bias Injection Attack, and Replay 
attack. This helps the intrusion with terrible ease and can lead to the failure of the detection system. Dos attack is an Associate in Nursing attack meant to wash up a machine or network, making the system inaccessible to its legitimate users which could lead to a holt inside the full network and increase in delay of knowledge transfer. DOS attacks accomplish this by flooding the targets with traffic or inflicting its info that triggers a crash. In an exceedingly Bias injection attack, Associate in Nursing assaulter gains access to frequency detector measurements and corrupts the information transferred to the automated generation management unit. This additionally ends up in a rise within the load of the network. Replay Attack is additionally referred to as playback attack the assaulter eavesdrops additionally as influences communication channels such communicated data is re-transmitted later. These three attacks will adversely affect the system and can lead to the failure of the system. To overcome and to attain higher accuracy and a less complex algorithm we have come up with a new solution to this. This is a modelling framework for the closed-loop system with the intrusion detection system, and a process procedure to style and cipher the intrusion detection system. This will reduce overfitting by regularization. Enhance the detection ability of IDS will be provided. This system will be based upon the LSTM algorithm.

\section{Existing System}

The expansion of remote organize activity assault characteristics has light-emitting diode to issues that old IDS methods with tall false-positive rate, low discovery potency, and destitute generalization capacity. To reinforce security and progress the location capacity of malicious interruption behaviour amid a remote arrange. To begin with, the organize activity data is characterized, pre-processed and at that point designed the arrange interruption traffic data by ICNN. The low-level intrusion activity data is conceptually portrayed as progressed alternatives by CNN, that extricated independently the test choices and optimizing arrange parameters by random SGD rule to meet the demonstrate. At long last, we tend to conduct sample that takes a look at to discover the interruption behaviour of the network. The recreation comes about appear that the strategy arranged in this paper has higher location precision and genuine positive rate beside a lesser false-positive rate. Then take a look at the outcomes about set KDDTest + in this paper that appears compared by various ordinary models, the location exactness is eight.

First, coaching is performed in bunches inside the network. Each coaching at irregular chooses a fixed-size piece as an input from pre-processed coaching data. The info parameter measurements entered all through coaching are the consequent (BatchSize1, H1, W1, Channel1) multidimensional parameter settings. For each coaching, a square of measure $\mathrm{M}$ is being chosen from the data. The top and measurement of an input file is being settled as one 122 severally, thus the channel remains single. The reason of backpropagation is iteratively alter weight and inclination of each layer's error between the specific yield worth and thus the perfect yield worth till the demonstrated model accomplishes a legitimate impact. Inside the strategy, to rapidly notice an optimum weight $\mathrm{W} 1$, bias B1 and thus the output $\mathrm{f1}(\mathrm{X})$ of the network will work according to coaching input $\mathrm{X}$ and loss operate $\mathrm{C}(\mathrm{W} 1, \mathrm{~B} 1)$ is prepared for finding an ideal combination of parameters to evaluate the degree of work. Random SGD run gives the easiest way to constrict this misfortune work. Forward propagation highlight extraction: The pre-processed coaching information is being forwarded as an input record, so the extraction will be performed by ICNN's independent wit. Backward Propagation Optimization: The strategy of mistake back propagation is conducted in keeping with the loss worth gotten by CNN coaching, Thus the parameters are optimized over and over till the show performs a legitimate joining affect.

\section{Proposed System}

This paper arranged a topology for a remote organized system that is considered underneath numerous cyber-attack outcomes, and a disseminated interruption discovery framework is implied to spot the presence of assaults. More particularly, this paper presents the modelling system for the closed-loop framework with the IDS, the machine method to fashion and calculate the IDS. This machine procedure conveys a steady closed-loop framework with the IDS being touchy to cyber-attacks. A re-enactment case is utilized as an illustration by applying the arranged method assist as its viability. In this paper, 3 assortments of cyber-attacks are tended to: (i) DoS assault, wherever a guilty party squares the transmission of knowledge between sending and accepting hubs; (ii) Replay assault, wherever a wrongdoer listens stealthily and records transmitted information, then replays an information at a afterward time;(iii) Bias Injection assault, wherever a wrongdoer infuses values into a transmitted information to oversee the knowledge. 


\subsection{Advantages}

When you perceive the association between the freelance and variable options a linear relationship, this formula is that the simplest to use owing to its less quality compared to different algorithms. Mathematically, it is easy to estimate the weights and you've got a guarantee to hunt out best weights. Over-fitting are reduced by regularization. Support mathematics approach. These organizations can gain from models apply them once a steady occasion emerges, making them ready to bear sum occasions. They will play out various assignments in equal while not contacting the framework execution. Generate new choices from restricted series of choices.

\subsection{Project Modules}

\subsubsection{Module 1: Exploratory Info Analysis}

Exploratory info analysis (EDA) is Related to Nursing approach/ philosophy for information investigation that utilizes an expansion of methods (generally graphical) to

1. To maximize knowledge into an information set.

2. To reveal fundamental structure.

3. To extricate crucial variables.

4. To identify exceptions and anomalies.

5. To test basic assumptions.

6. To create penurious models.

7. To decide ideal issue settings.

EDA isn't a twin of applied math graphics though the 2 terms square measure used nearly interchangeably. applied math graphics square measure a set of techniques-all diagrammatically based mostly and every one specializing in one information characterization fact. EDA includes a bigger setting; EDA is a perspective to data investigation that delays similar ancient suspicions with respect to what reasonably show the data take after with the extra direct approach of allowing the data itself to uncover its basic structure and demonstrate model. EDA isn't a simple collection of procedures; EDA may be a reasoning on be that as it may we tend to dismember an information set; what we look for; however, we tend to see; and the way we tend to translate.

\subsubsection{Module 2: Feature Selection}

Labels represent string labels of each ordinal and nominal options to the specific feature set. Some labels could have orders related to them (ordinal features) whereas others might not have any orders related to them (nominal features). it's a very important a part of information preprocessing to code labels befittingly in numerical kind to form certain that the training algorithmic program interprets the options properly. within the following section, you may see however you'll use LabelEncoder category of sklearn. Preprocessing module to code labels of categorical options. Label encryption refers to remodeling word labels into a numerical kind so algorithms will perceive the way to treat them. Statistics show that the amount of traffic packets in most flows is a smaller amount than ten, however the amount of traffic packets in some flows is larger than ten or perhaps exceeds a hundred. Since the payload length of every traffic packet isn't equal, to use our information to coach our classification model, we tend to solely extract one hundred sixty bytes in every traffic packet because the traffic packet options. Therefore, if the packet length of a packet is a smaller amount than one hundred sixty bytes, then we want to use zero paddings for this packet. If a packet is longer than one hundred sixty bytes, we tend to solely take the primary one hundred sixty bytes. to form the info sent to the model has an equivalent dimensions, we tend to solely use the primary ten traffic packets of every flow.

\subsubsection{Module 3: Prediction}

The train-test split technique is reasonable once you have a truly monster dataset, a costly model to mentor, or need a legitimate gauge of model execution rapidly. The method includes taking a dataset and isolating it into 2 subsets. the essential set is utilized to suit the model and is referred to in view of the instructing dataset. The subsequent set isn't wont to prepare the model; all things being equal, the info part of the dataset is given to the 
model, at that point forecasts are made and contrasted with the normal qualities. This second dataset is referred to due to the check dataset.

Train Dataset: To coordinate with the AI model.

Test Dataset: To assess the match AI model.

The goal is to assess the presence of the AI model on new data: information not want to prepare the model. of course, the program disregards the principal request of information. It at irregular picks data to make the training and check set, which is normally a captivating element in true applications to stay away from potential curios existing inside the data readiness technique. To debilitate this element, just set the mixed boundary as False $($ default $=$ True $)$. The LSTM model can get familiar with a presentation that maps a grouping of past perceptions as a contribution to relating yield perception. All things considered, the succession of perceptions ought to be redesigned into numerous models from that the LSTM will learn. A square has parts that make it more astute than an old-style nerve cell and a memory for ongoing arrangements. A square works upon a partner input succession and each entryway inside a square uses the sigmoid enactment units to manage whether they are set off or not, making the adjustment of state and expansion of information moving through the square restrictive.

\subsection{Algorithms Used}

1. LSTM (Long memory) - Long short Memory (LSTM) might be a style of a persistent neural organization fit for learning request reliance in grouping expectation issues. It can be a conduct required in cutting edge disadvantage spaces like man-made reasoning, discourse acknowledgment, and that's just the beginning. Intermittent organizations had an inside express which may address setting information. They keep information worried past contributions of an amount of your time that is not mounted on an earlier. In any case, rather relies upon its loads and furthermore the PC document.

2. Random Forest- It is a well-known AI calculation that has a place with the directed learning procedure. Irregular Forest is a classifier that contains various choice trees on different subsets of the given dataset and takes the normal to improve the prescient precision of that dataset. The precision of the Extra Trees Classifier is 98.66\%.

3. The Neural Network-It is a progression of calculations that tries to perceive basic connections in a bunch of information through an interaction that impersonates the manner in which the human mind works. In this sense, neural organizations allude to frameworks of neurons, either natural or counterfeit in nature. The precision of Neural Network is $91.52 \%$.

\section{Results and Discussions}

Vectorization is employed to hurry up the Python code while not employing a loop. Vectorization such a operate will facilitate in minimizing the time period of code with efficiency. varied operations square measure being performed over vector like inner product of vectors that is additionally referred to as a real number because it produces one output, outer product which ends in an exceedingly matrix of dimension up to length $\mathrm{X}$ length of the vectors, component wise multiplication that product the component of same indexes and dimension of the matrix stay unchanged. For pooling, we have a tendency to use the Pool category. once one will produce a pool of processes which will carry the total tasks submitted to that. A pool object controls a pool of worker to choose that job area unit typically submitted and it supports asynchronous result that has timeouts, callbacks, and a parallel map implementation. CPU-count() is employed within the method is none and initializer(*initargs) this operate calls once the initializer isn't any. In logical control, consideration is that the intellectual activity of by choice focusing on one a few things though overlooking others. A neural organization is considered to be an endeavor to impersonate human bandeauin activities in a really improved on way. Consideration Mechanism is furthermore an undertaking to carry out indistinguishable activity of by determination focusing two or three significant things while overlooking others in profound neural organizations. For instance, Let's just say you are looking a gaggle pic of the first personnel. Regularly, there will be gaggle of young people sitting across numerous columns, thus the instructor can sit some place in the middle. Presently, on the off chance that anybody poses the inquiry, "What number of people square measure there?", anyway would you be able to respond to it? Essentially by examination heads, isn't that so? You don't have the opportunity to consider different things inside the pic. Presently, on the off chance that anybody poses a particular inquiry, "Who is that the instructor inside the photograph"? It'll only start attempting to discover the alternatives of partner degree grown-up inside the pic. the rest of the choices can just be unnoticed. this is 
regularly the 'Consideration' that our cerebrum is staggeringly capable at carrying out. The consideration component arose as partner degree improvement over the encoder decoder-based neural MT framework in etymological correspondence measure (NLP). Afterward, this system, or its variations, was utilized in elective applications, along with PC vision, discourse measure, and so on. Both the encoder, decoder square measure piles of LSTM/RNN units. That works inside 2 after advances:

1. The code LSTM is utilized to strategy the entire information sentence and encode it into a setting vector, that will be that the last secret condition of the LSTM/RNN. this is frequently expected to be a legit layout of the info sentence. Every one of the moderate conditions of the encoder square measure unnoticed, and in this way the last state is intended to be the underlying secret condition of the decoder.

2. The decoder LSTM or RNN units turn out the words in an incredibly sentence one when another. To put it plainly, there square measure 2 RNNs/LSTMs. Once we tend to choose the encoder - this peruses the info sentence and attempts to shape feeling of it, prior to summing up it. It passes the diagram (setting vector) to the decoder that deciphers the info sentence by basically seeing it. TP: 83484, FP: 115, TN: 983464, FN: 14330 Overall Accuracy is $91.86 \%$.

\subsection{Figures and Tables}

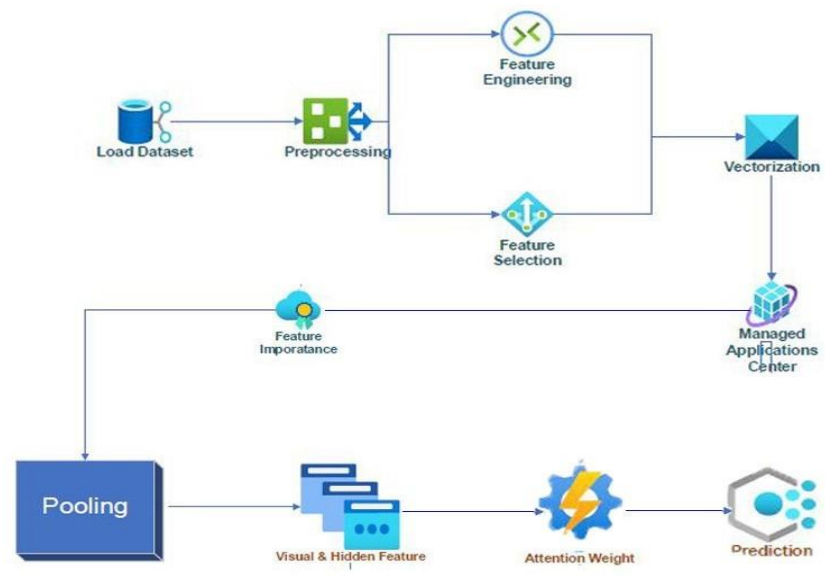

Figure 1: Architecture Diagram

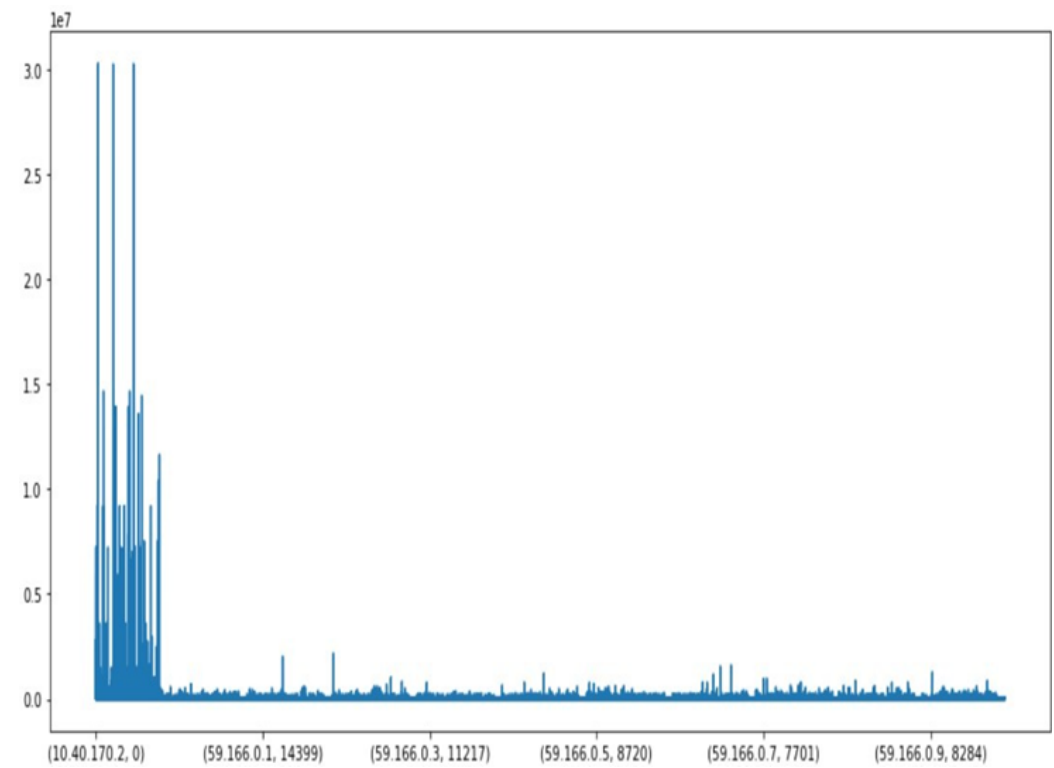

Figure 2: Dataset-1 


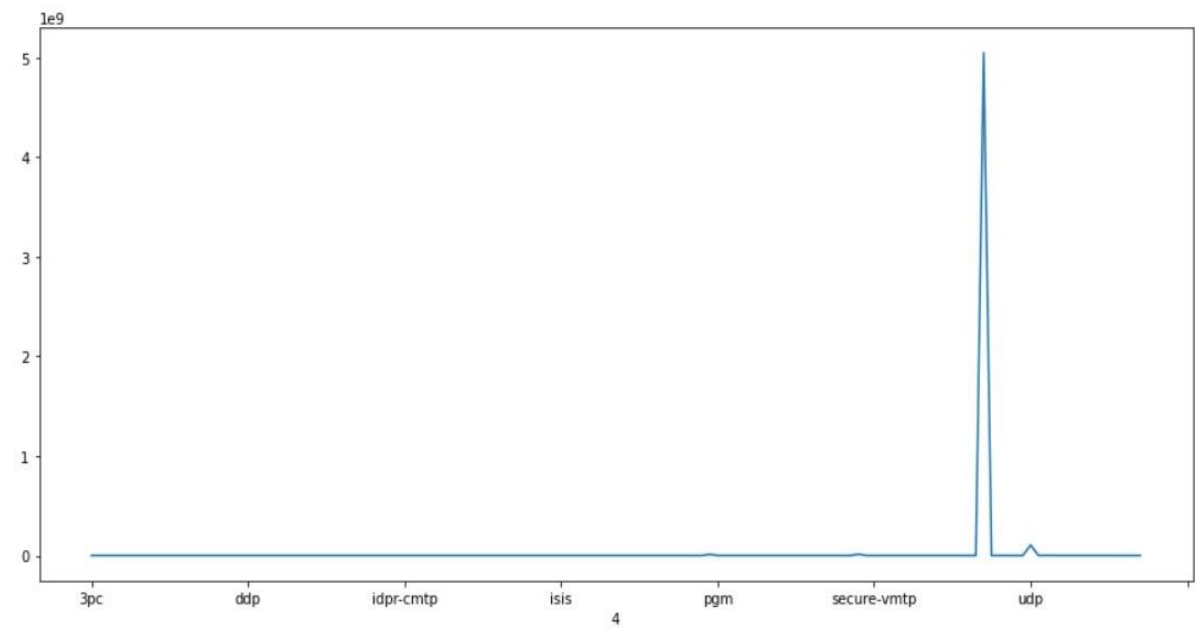

Figure 3: Dataset-2

Feature Importance Neural Network -

$\mathrm{X}_{\mathrm{n}} \mathrm{n}_{\mathrm{t}}$ rain $=\mathrm{np}$.concatenate $\left(\left(\mathrm{Y}_{\mathrm{n}} \mathrm{n}_{\mathrm{At}} \mathrm{rain}\left[:, \mathrm{np}\right.\right.\right.$.newaxis], $\mathrm{X}_{\mathrm{n}} \mathrm{n}_{\mathrm{t}}$ rain $)$, axis $=$ $\mathrm{nn}_{\mathrm{i}} \mathrm{mp}, \mathrm{nn}_{\mathrm{n}}$ dices, $\mathrm{nn}_{\mathrm{s}} \mathrm{td}=$ find $_{\mathrm{i}} \mathrm{mportances}\left(\mathrm{X}_{\mathrm{n}} \mathrm{n}_{\mathrm{t}}\right.$ rain, $\mathrm{Y}_{\mathrm{n}} \mathrm{n}_{\mathrm{t}}$ rain $)$ plot $_{\mathbf{f}}$ eature ${ }_{\mathrm{i}}$ mportances $\left(\mathbf{X}_{\mathrm{n}} \mathrm{n}_{\mathrm{t}}\right.$ rain, $\mathrm{nn}_{\mathrm{i}}$ mportances, $\mathrm{nn}_{\mathrm{i}}$ ndices, $\mathrm{nn}_{\mathrm{s}} \mathrm{td}$, title $=^{\prime}$ Featureimportances(Neuralnetwork $)^{\prime}$ )

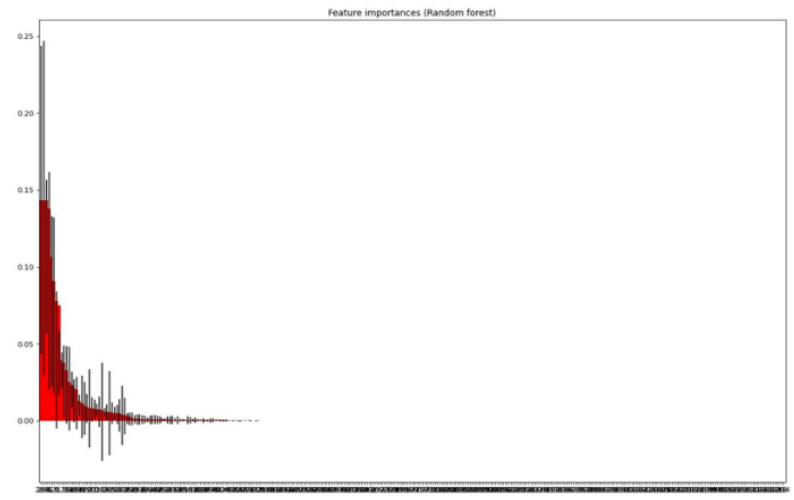

Figure 4: Random Forest

Figure-4 demonstrates the element significances of Random Forest and has plots in particular (X-rf-train, rfimportances, rf-indices, rf-std).

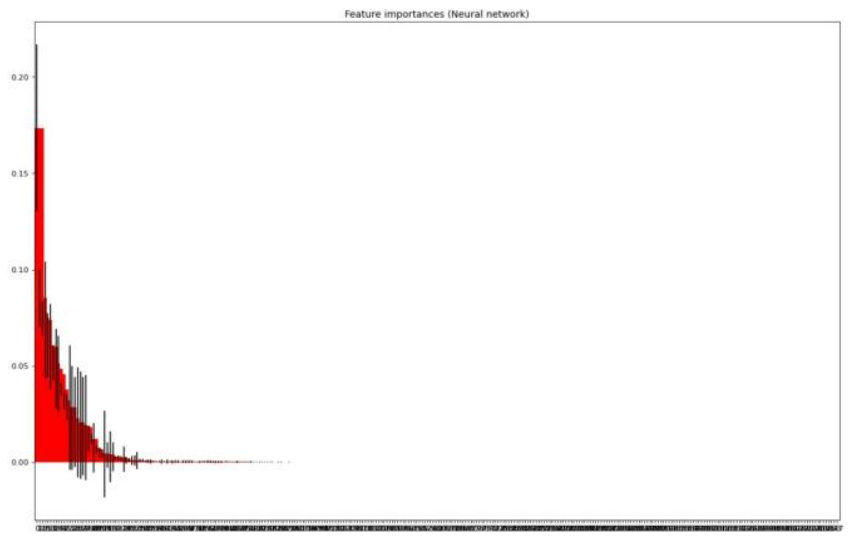

Figure 5: Neural Network 
Figure-5 demonstrates the element significances of Random Forest and has plots in particular (X-nn-train,nnimportances, nn-indices, nn-std).

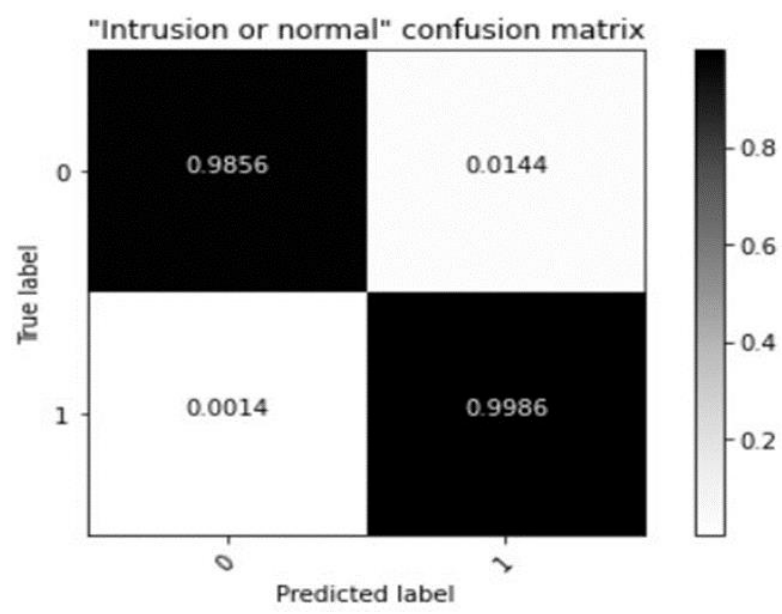

Figure 6: Intrusion or Normal Confusion Matrix

Figure-6 indicates of the True Positive, False Negative, True Negative, False Positive accuracy rates in an Intrusion Confusion Matrix form..
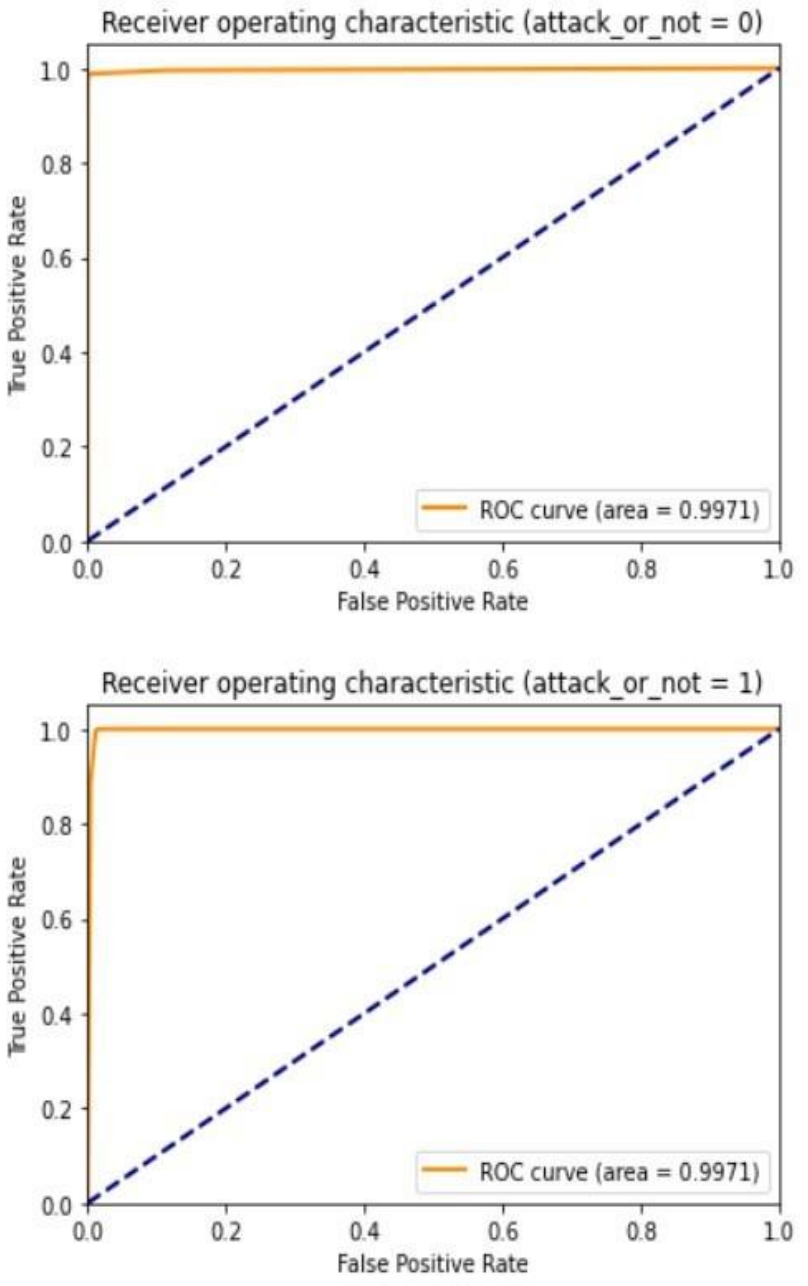

Figure 7: False Positive Rates 
The accuracy value is directly proportional to the epoch value. Similarly, the loss value is inversely proportional to the epoch value.

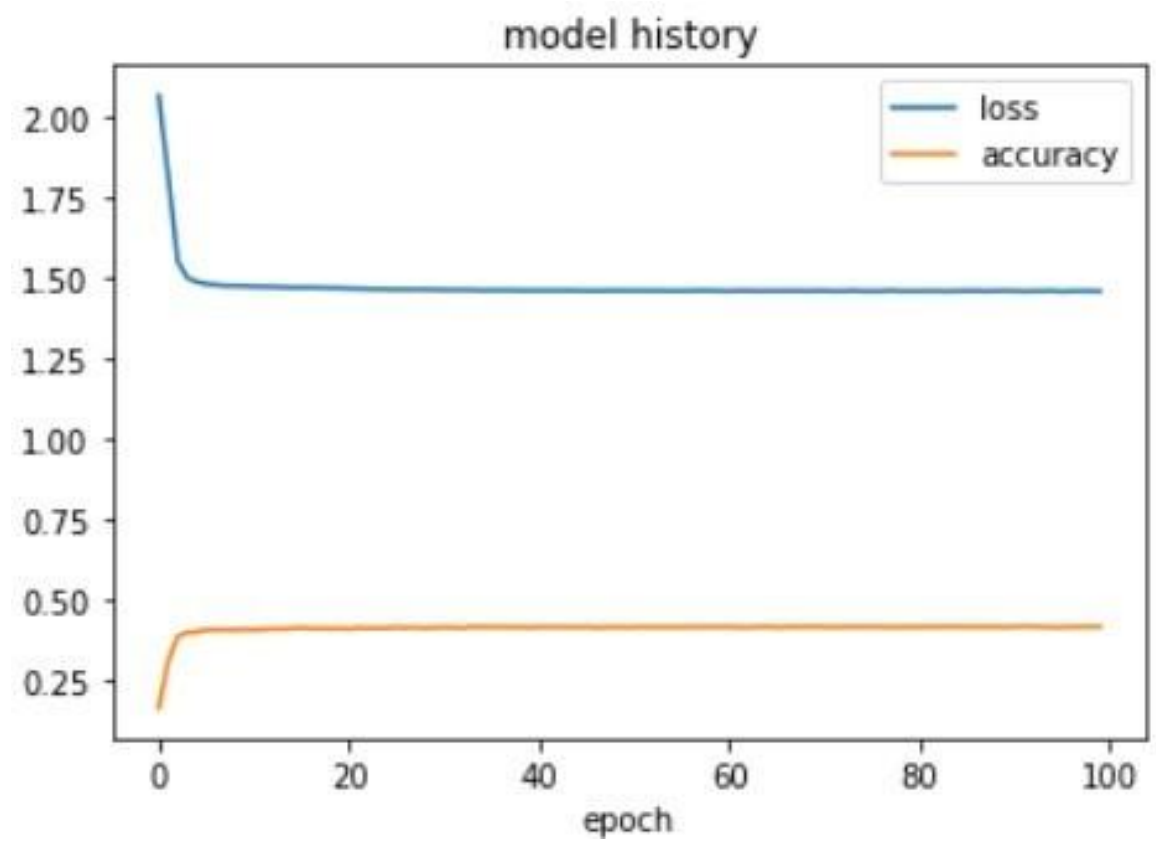

Figure 8: Model History

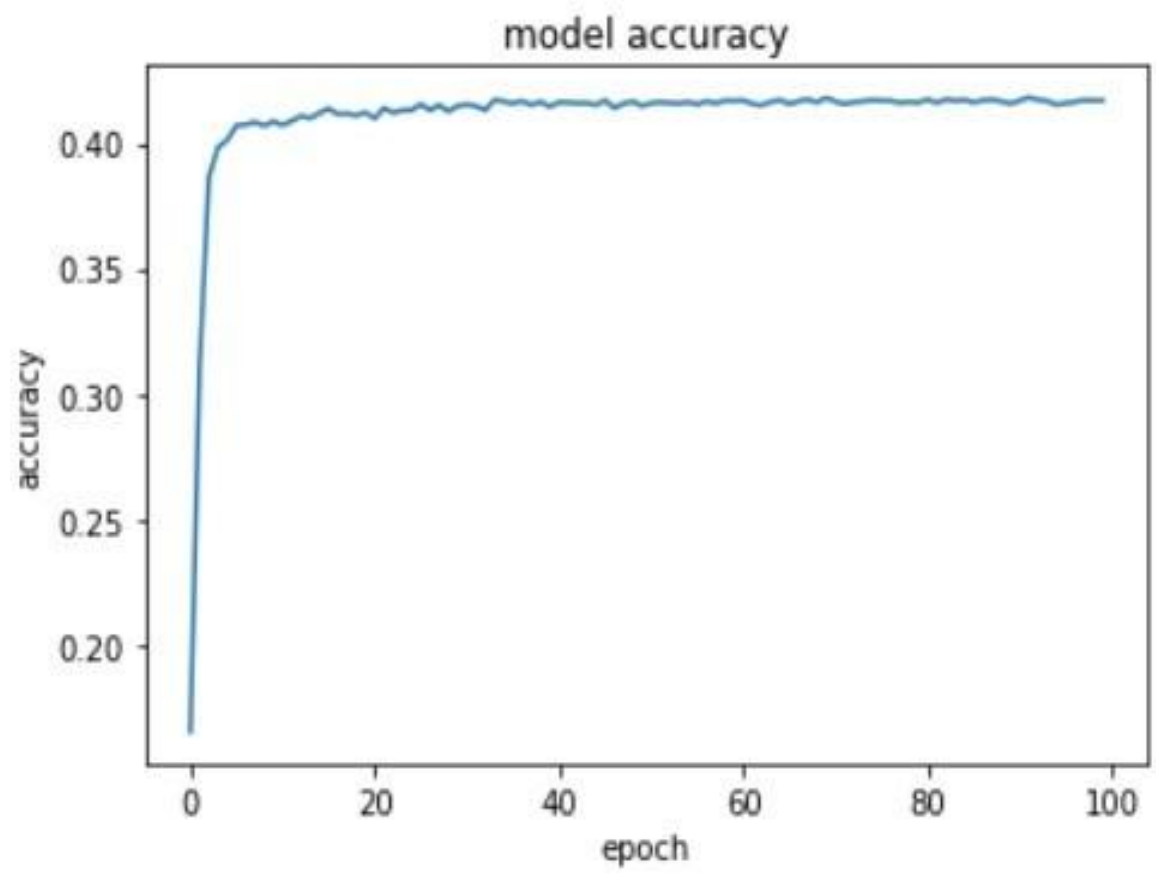

Figure 9: Model Precision 


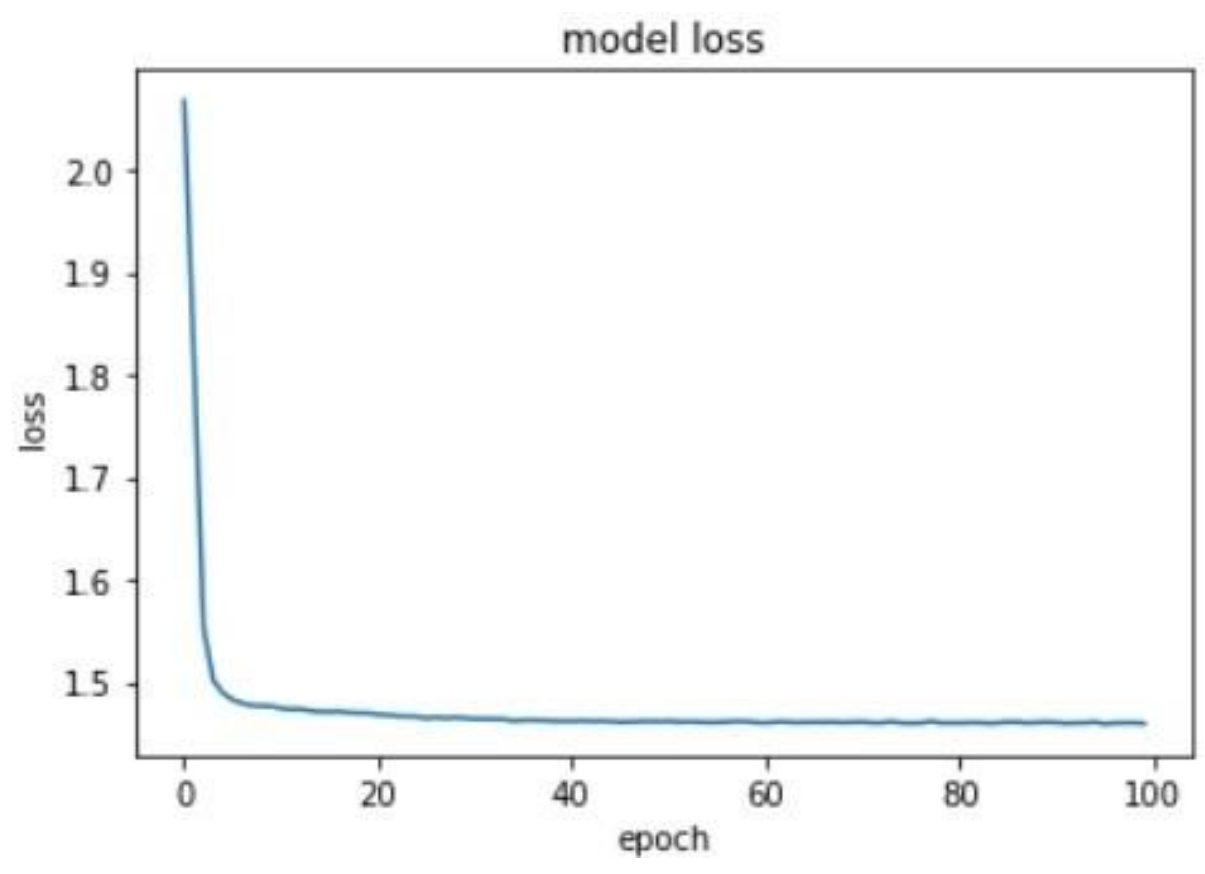

Figure 10: Model Dropping

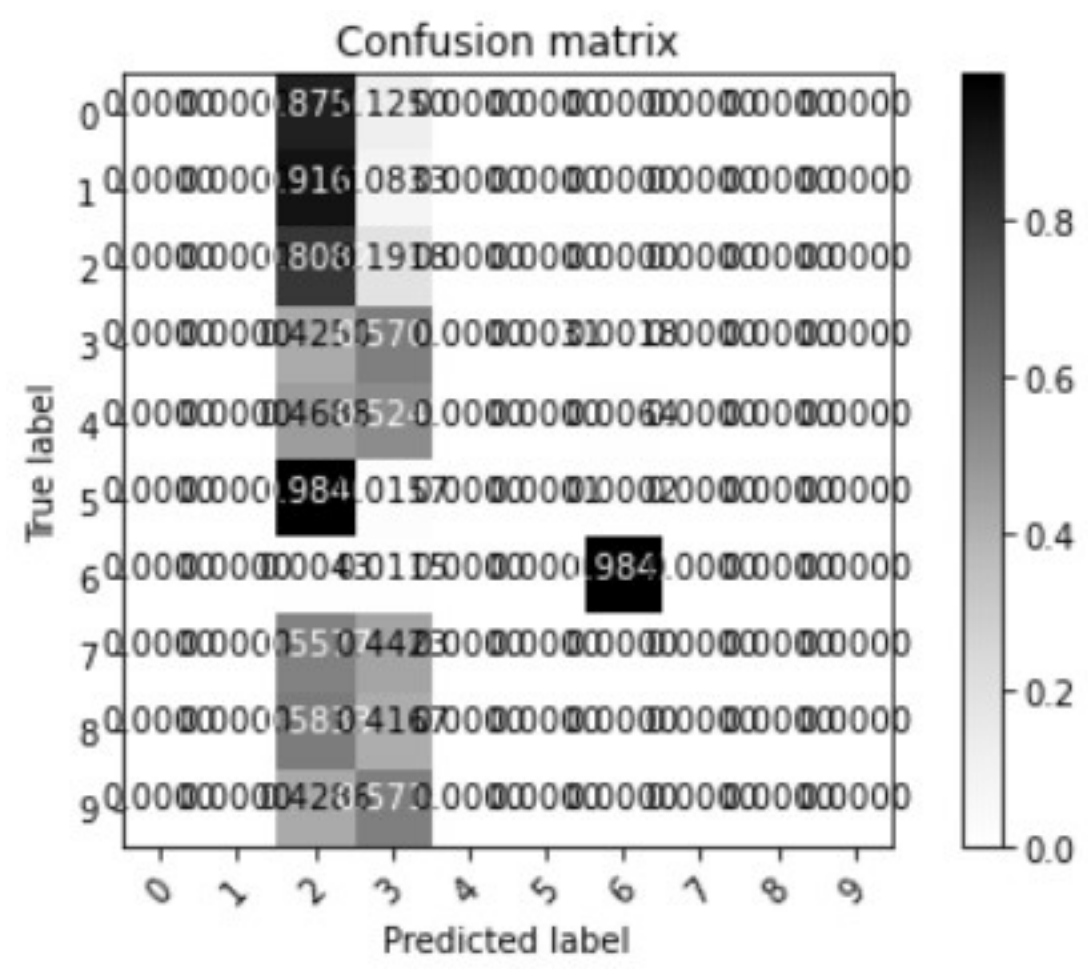

Figure 11: Confusion Matrix 


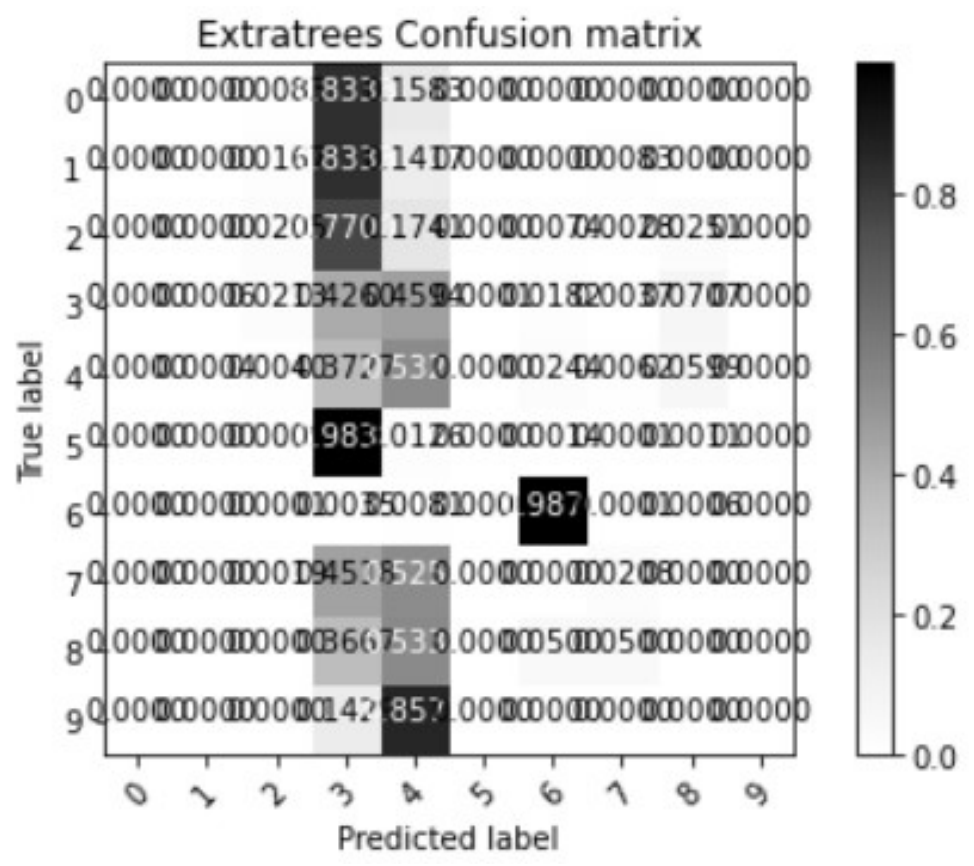

Figure 12: Extra-Trees Confusion Matrix

\subsection{Implementation}

In this dataset comprising of many values and expressions would be used to train our model and analyses the threats that the present system is prone to. It reads files which contains all the required information for the same. The data is read and fetched from the csv files using the "read-csv" command and it reads all the features info. Then it assigns all the fields the machine requires and assigns all the necessary values to it. Then we assign all the data info and data values and feature types. In next step the columns of the indices for the feature types are plotted and the csv files are read. Once the data is fetched and read, it is concatenated and a set of 10 rows and 49 columns are fetched. Then the Pre-processing of data is done and conversion of data takes place. In this step data is pre-processed and transformed the raw data into understandable and readable format.

Next the not available values are replaced and made ready for the further process. Further the stripping of the nominal columns and setting them to lower case is done. Then the targets are changed and set. In the next step the data sets are sliced so as to achieve the correct level of proper analysis. The process of replacing the NA's is done after normalization and concatenation of the $\mathrm{x}$ and $\mathrm{y}$ axis so as to get a perfect set of data. Then $\mathrm{X}$ is split to test and train the datasets. Once this is done, the feature importance is traced with Extra Trees Classifier. The feature importance is plotted using the Random Forest and Neural network. The datasets are tested multiple times to check for the accuracy. The curves are plotted and checked for the accuracy. After the tests the confusion matrix are plotted so as to gain the predicted table. After this the neural network is created and trained which is then plotted using training history data with loss and accuracy as their values. Again, the final test is run and the confusion matrix once more is plotted so as to get the final matrix for the same with higher accuracy rate. And hence the final result is plotted in the matrix.

\section{Conclusion}

IDS is a significant focal point of examination in network security. This paper investigates the Neural Networks techniques being utilized in IDS. An interruption identification framework with LSTM network is introduced. This paper proposes another oddity based way to deal with identify and forestall wormhole and surging assaults in impromptu organizations. The proposed arrangement depends on various levelled design and uses a measurable methodology. The interruption recognition and counteraction framework was executed in group heads. The focal point of future exploration is on the best way to improve the identification exactness of the location model. 


\section{References}

[1]. Hongyu Yang, Fengyan Wang. (2019). Wireless Network Intrusion Detection Based on Improved Convolutional Neural Network. IEEE Access, Volume 7.

[2]. Zhiguan Hu, Liejun Wang, Lei Qi, Yongming Li, Wenzhong Yang. (2020). A Novel Wireless Network Intrusion Detection Method Based on Adaptive Synthetic Sampling and an Improved Convolutional Neural Network, IEEE Access, Volume 8.

[3]. Aechan Kim, Mohyun Park, Dong Hoon Lee. (2020). AI-IDS: Application of Deep Learning to RealTime Web Intrusion Detection, IEEE Access, Volume 8.

[4]. Liqun Yang, Liang Yin, Zhoujun Li. (2020). Real-Time Intrusion Detection in Wireless Network: A Deep Learning-Based Intelligent Mechanism, IEEE Access, Volume 8.

[5]. Wenming Wang, Haiping Huang, Qi Li, Fan He, Chao Sha. (2020). Generalized Intrusion Detection Mechanism for Empowered Intruders in Wireless Sensor Networks, IEEE Access, Volume 8.

[6]. Shuai Jiag, Juan Zhao, Xiaolong Xu. (2020). SLGBM: An Intrusion Detection Mechanism for Wireless Sensor Networks in Smart Environments, IEEE Access, Volume 8.

[7]. Christian Miranda, Georges Kaddoum, Elias Bou-Harb, Sahil Garg. (2020). A Collaborative Security Framework for Software Defined Wireless Sensor Networks. IEEE Transactions on Information Forensics and Security, Volume 15.

[8]. Yuxiang Lin, Yi Gao, Bingji Li, Wei Dong. (2020). Revisiting Indoor Intrusion Detection with WiFi Signals: Do Not Panic Over a Pet, IEEE Internet of Things Journal, Volume 7.

[9]. Zhuo Chen, Na Lv, Pengfei Liu, Yu Fang, Kun Chen, Wu Pan. (2020). Intrusion Detection for Wireless Edge Networks Based on Federated Learning, IEEE Access, Volume 8.

[10]. Reza Parsamehr. (2020). IDLP: An Efficient Intrusion Detection and Location-Aware Prevention Mechanism for Network Coding-Enabled Mobile Small Cells, IEEE.

[11]. Sumit Pundir, Mohammad Wazid, Devesh Pratap Singh, Ashok Kumar Das. (2019). Intrusion Detection Protocols in Wireless Sensor Networks Integrated to Internet of Things Deployment: Survey and Future Challenges, IEEE Access, Volume 8.

[12]. Mohammad Shabaz Hussan, Khallel Ur Rahman Khan. (2020). Network-Based Anomaly Intrusion Detection System in MANETS, Fourth International Conference on Inventive Systems and Control.

[13]. Markus Hanselmann, Thilo Strauss, Katharina Dormann, Holger Ulmer. (2020). CANet: An Unsupervised Intrusion Detection System for High Dimensional CAN Bus Data, IEEE Access, Volume 8.

[14]. Md Delwar Hossain, Hiroyuki Inoue. (2020). LSTM-Based Intrusion Detection System for InVehicle CAN Bus Communications, IEEE Access, Volume 8.

[15]. R. Vijayanand, D. Devaraj. (2020). A Novel Feature Selection Method Using Whale Optimization Algorithm and Genetic Operators for Intrusion Detection System in Wireless Mesh Network, IEEE Access, Volume 8.

[16]. Jing XU, Shimin Gong, Yuze Zou. (2019). Redundant Sniffer Deployment for Multi-Channel Wireless Network Forensics with Unreliable Conditions, IEEE Transactions on Cognitive Communications and Networking, Volume 6.

[17]. Jose Ribeiro, Firooz B. Saghezchi, Georgios Mantas. (2020). HIDROID: Prototyping a Behavioral Host-Based Intrusion Detection and Prevention System for Android, IEEE Access, Volume 8.

[18]. Satya Katragadda, Paul J. Darby, Andrew Roche. (2020). Detecting Low-Rate Replay-Based Injection Attacks on In-Vehicle Networks, IEEE.

[19]. Anurag Busha, Vakeesh Kanna, Sagar Naidu. (2019). Network Analysis of Intrusion Detection Based on Machine Learning and Deep Learning, International Journal of Engineering and Advanced Technology, Volume 8.

[20]. Geethapriya Thamilarasu. (2020). An Intrusion Detection System for Internet of Medical Things, IEEE Access, Volume 8. 